\title{
Genetic Variants of Hemophilia B: Detection by Means of a Specific PTC Inhibitor
}

\author{
Harold R. Roberts, James E. Grizzle, William D. McLester, and \\ George D. Penick \\ From the Departments of Pathology and Medicine, School of Medicine, \\ University of North Carolina, Chapel Hill, North Carolina
}

A BS T R $\Lambda$ C T Jemophilia $\mathrm{S}$ can be divided into at least two mutant forms different from the mild, moderate, and severe categories previously describer. In about $90 \%$ of hemophilia B patients. PTC-inhibitor-neutralizing activity is reduced in proportion to PTC clotting activity. In about $10 \%$ of the patients, PTC-inhibitor-neutralizing activity is fully effective, whereas PTC clotting activity. is reduced. Extensive pedigree studies indlicate that the presence or absence of inhibitor-neutralizing activity is genetically determined. It is suggested that those hemophilia $B$ mutants with clecreased inhibitor-neutralizing material produce decreased amounts of PTC-protein. It is further suggested that those with normal levels of inhibitor-nentralizing material produce normal amounts of PTC-protein, which is stru^turally altered so as to lose procoagulant activity but which retains inhibitor-neutralizing activity. The latter group may be analogous to $\mathrm{CRN}^{+}$mutants described it bacteria and Nourospora.

\section{INTRODUCTION}

We previously described a circulating anticoagulant which specifically inhibited PTC (plasina thromboplastin component. Factor IX. Christmas

Dr. James E. Grizzle's present address is the School of Public Health, Dept. of Biostatistics, University of North Carolina, Chapel Hill, N. C. 27514. Address requests for reprints to Dr. Harold R. Roberts, Departments of Pathology and Nedicine, University of North Carolina School of Medicine, Chapel Hill, N. C. 27514.

Reccived for publication 31 Jul! 196 i and in reicisid form 22 Siptimber 1967. factor) $(1,2)$. Properties of this inhibitor strongly suggested that it was an antibody against PTC (3). This type of inhibitor can be neutralized by the PTC of normal plasma and, according to Fantl et al., by PTC which is altered so as to retain inhibitor-neutralizing activity but not clotting activity (4).

Previous observations have shown that plasma from most severely affected hemophilia B patients does not contain material capable of neutralizing a specific PTC-inhibitor $(2,4)$. On the other hand, a single patient whose plasma did contain inhibitor-neutralizing activity has been reported (4). These observations suggested that two types of hemophilia B might exist. To examine this possibility a comparison of the PTC clotting and inhibitor-neutralizing activities in plasma from a large number of hemophilia $\mathrm{B}$ patients was made. Our results slow that in $90 \%$ of patients, inhibitor-neutralizing activity is reduced in proportion to the PTC clotting deficiency. In the remaining $10 \%$ of patients, inhibitor-neutralizing activity is fully effective whereas PTC clotting activity is clearly reduced. These observations are interpreted to mean that the PTC deficiency in most patients represents an actual decrease in PTCprotein, whereas in other patients it reflects the presence of an altered PTC-protein which retains inhibitor-neutralizing activity, but which loses clotting activity.

\section{METHODS}

PTC-dificient subiects. For this study PTC-deficient subjects were shown to have the defect by laboratory testing in the Clinical Coagulation Laboratory at the 
University of North Carolina. 62 affected persons from 25 pedigrees were restudied for inhibitor-neutralizing activity.

All affected patients within each family had sımilar PTC levels and similar symptoms of bleeding, and each family could be said to have either a severe, moderate, or mild bleeding disorder. In general, patients were considered to be severely affected when there was evidence of repeated and spontaneous hemarthroses resulting in chronic joint disease : mild bleeders were those with a history of bleeding only after surgical or other forms of trauma, and moderate bleeders were those with intermediate symptoms. The mothers of the PTC-deficient patients described in this paper were regarded as carriers for PTC deficiency.

Normal subjects were laboratory personnel, medical students, and unaffected relatives of PTC-deficient patients. PTC levels in this group varied from 86 to $140 \%$ when assayed against pooled plasma from 10 other normal individuals.

Plasma from all patients was prepared from blood drawn into either $3.2 \%$ sodium citrate (1 part to 8 parts of blood) or $0.1 \mathrm{M}$ sodium oxalate (1 part to 9 parts blood), using the two-syringe technique. The blood was then centrifuged in a Servall angle centrifuge at $3,000 \mathrm{~g}$ for $10 \mathrm{~min}$. Plasma uas aspirated with glass pipettes, placed in plastic tubes, and stored at $-20^{\circ} \mathrm{C}$ for no longer than 7 days. $\mathrm{BaSO}_{4}$-adsorbed plasma was prepared by adsorbing $1 \mathrm{ml}$ of oxalated plasma with $100 \mathrm{mg}$ of $\mathrm{BaSO}_{4}$ at $5^{\circ} \mathrm{C}$ for $30 \mathrm{~min}$.

Coagulation studics consisted of the following: partial thromboplastin time tests (5); and specific assays for AHF [antihemophilic factor, Factor VIII (5); Stuart factor, Factor X (6); and Hageman factor, Factor XII (7) ]. PTC was assayed by a method similar to that described by Barrow, Bullock, and Graham (8). Briefly, this procedure consisted of a comparison of partial thromboplastin times of various dilutions of control and test plasmas. Plasma dilutions of $10,5,2.5$, and $1.25 \%$ were made serially with buffered, citrated saline. Onehalf $\mathrm{ml}$ of each dilution of plasma was mixed, in sequence, with $0.5 \mathrm{ml}$ of PTC-deficient plasma in a glass tube containing $10 \mathrm{mg}$ of kaolin (Fisher Scientific Co., Fair Lawn, N. J.). The tubes were covered with parafilm, incubated at $28^{\circ} \mathrm{C}$ for $15 \mathrm{~min}$ (with frequent mixing), and then placed in a melting ice bath until tested. Twotenths $\mathrm{ml}$ of each of the above mixtures was placed, in sequence, into clean $10 \times 75 \mathrm{~mm}$ glass tubes, incubated at $37^{\circ} \mathrm{C}$ for $60 \mathrm{sec}$, then recalcified with $0.2 \mathrm{ml}$ of a solution containing 1 part of $1: 10$ dilution of cephalin (Thrombofax, Ortho Pharmaceuticals, Raritan, N. J.) and 1 part of calcium-imidazole-saline (8) which had been prewarmed to $37^{\circ} \mathrm{C}$. The clotting times on all plasma dilutions were determined in duplicate at $37^{\circ} \mathrm{C}$, averaged, and plotted against plasma dilution on semilogarithmic paper. PTC assays were performed on all tested patients at least three times.

Inhibitor source was plasma from a patient with hemophilia B who had developed a circulating anticoagulant that specifically inhibited PTC. Characteristics of this inhibitor, including those suggesting that the inhibitor is an anti-PTC antibody, have been described in detail previously (1-3). The same lot and concentration of inhibitor plasma was used in all experiments to be reported.

Inhibitor-neutralization tests were performed as shown in the flow chart of Fig. 1. The standard inhibitor concentration was prepared by diluting 1 part inhibitor plasma with 7.5 parts of citrated-imidazole-saline buffer ( $\mathrm{pH}$ 7.2). One-tenth $\mathrm{ml}$ of this dilution of inhibitor plasma was mixed with $0.9 \mathrm{ml}$ of the plasma to be tested (Mixture 1). In Mixture 1, both PTC and inhibitor are neutralized depending on the respective concentrations. PTC assays were then performed on an aliquot of the mixture after $20 \mathrm{~min}$ incubation at $28^{\circ} \mathrm{C}$ as a check on inhibitor concentration. The amount of inhibitor neutralized in Mixture 1 was determined by incubating one part of this first incubation mixture with one part of normal plasma as a source of PTC (Mixture 2). Should significant inhibitor remain in the first incubation mixture, the residual $\mathrm{PTC}$ in the second incubation mixture would be considerably less than $50 \%$. Examples of inhibitor-neutralization tests obtained on normal plasma, PTC-deficient plasma, $\mathrm{BaSO}_{4}$-adsorbed plasma, etc. are shown in Table 1. The normal plasma control shows that the amount of PTC in normal plasma would neutralize virtually all of the inhibitor in Mixture 1, whereas the BaSO4-adsorbed normal plasma control suggests that complete absence of PTC in Mixture 1 should leave

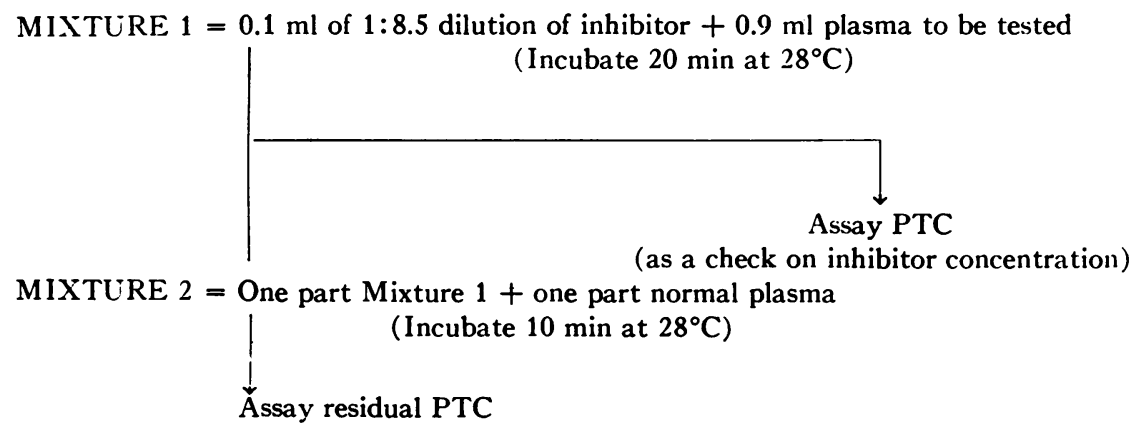

FIGURE 1 Technique demonstrating inhibitor neutralization. Residual PTC is directly proportional to the amount of inhibitor neutralized in first incubation mixture; therefore, it is a measure of the inhibitor-neutralizing material present in the test plasma. 
enough inhibitor to reduce the PTC level in Mixture 2 to about $5 \%$ of normal.

\section{RESULTS}

The data are summarized in Fig. 2 which is a $\log -\log$ plot of the PTC level of plasma (L-PTC) on the abscissa vs. the level of inhibitor-neutralizing activity (expressed as the log of residual PTC in Mixture 2 [LR-PTC]) on the ordinate. Each point in Fig. 2 represents the mean for the affected members of a single kindred. Roman numerals identify the different kindred, $\mathrm{C}$ denotes carriers, and the number is parentheses represents the number of affected persons studied in the kindred. The best-fitting straight line was calculated for these points, omitting the four kindred suspected of being "outliers" (described later). The method of weighted least squares was used, the weights being the number of affected individuals used in determining each point. The relationship between LR-PTC and L-PTC is described ade- quately by a straight line. With the exception of the "outliers," $95 \%$ of the variation in LR-PTC can be accounted for by the variation in L-PTC. Thus, the value of LR-PTC (inhibitor-neutralizing activity), predicted from a specified value of L-PTC, can be compared with its observed value by determining whether the point falls within the $99 \%$ confidence bands.

It can be seen that the plasma PTC levels in the various hemophilia $B$ pedigrees range from virtually undetectable amounts to levels overlapping the PTC concentrations found in hemophilia $B$ carriers. These findings reflect the various degrees of clinical severity of hemophilia B, known to range from mild to severe. Analysis of variance clearly demonstrates that important variation in plasma PTC levels is between, not within, kindred; i.e., all affected patients in a single kindred are of the same degree of severity. The within-family variance (mean square, 0.02607) was much smaller than the between-family vari-

TABLE I

Examples of Inhibitor Neutralization

\begin{tabular}{|c|c|c|c|c|c|c|}
\hline \multirow{3}{*}{ Type plasma or control } & \multirow{3}{*}{$\begin{array}{c}\text { Initial PTC } \\
\text { level }\end{array}$} & \multirow{3}{*}{$\begin{array}{l}\text { Inhibitor } \\
\text { dilutionf }\end{array}$} & \multicolumn{4}{|c|}{ Residual PTC (\%) } \\
\hline & & & \multicolumn{2}{|c|}{$\begin{array}{l}\text { First incubation } \\
\text { (Mixture 1) }\end{array}$} & \multicolumn{2}{|c|}{$\begin{array}{l}\text { Second incubation* } \\
\text { (Mixture 2) }\end{array}$} \\
\hline & & & Expected $\%$ & Observed \% & Expecled $\% \S$ & Observed \% \\
\hline Normal & 86 & $1: 85$ & $\sim 5$ & 3.8 & 52 & 50 \\
\hline Normal & 108 & $1: 85$ & $\sim 5$ & 2.6 & 51 & 63 \\
\hline Normal & 100 & $1: 85$ & $\sim 5$ & 5.9 & 53 & 36 \\
\hline Normal & 125 & $1: 85$ & $\sim 5$ & 3.0 & 51 & 47 \\
\hline Normal & 93 & $1: 85$ & $\sim 5$ & 1.0 & 55 & 51 \\
\hline PTC deficient & $<1$ & $1: 85$ & 0 & 0 & $\sim 50$ & 3.8 \\
\hline PTC deficient & $<1$ & $1: 85$ & 0 & () & $\sim 50$ & 3.9 \\
\hline PTC deficient & $<1$ & $1: 85$ & 0 & 0) & $\sim 50$ & 7.1 \\
\hline PTC deficient & $<1$ & $1: 85$ & 0 & 0) & $\sim 50$ & 2.6 \\
\hline PTC deficient & 11 & $1: 85$ & 0 & 0 & $\sim 50$ & 16.5 \\
\hline PTC deficient & 3 & $1: 85$ & 0 & 0 & $\sim 50$ & 4.0 \\
\hline PTC deficient & 5 & $1: 85$ & 0 & 0 & $\sim 50$ & 5.0 \\
\hline PTC deficient & 4 & $1: 85$ & 0 & ) & $\sim 50$ & 10.0 \\
\hline $\mathrm{BaSO}_{4}$-adsorbed normal plasma & 0 & $1: 85$ & 0 & 0 & $\sim 50$ & 5.7 \\
\hline $\mathrm{BaSO}_{4}$-adsorbed normal plasma & 0 & $1: 85$ & 0 & () & $\sim 50$ & 1.9 \\
\hline $\mathrm{BaSO}_{4}$-adsorbed normal plasma & 0 & $1: 85$ & 0 & ) & $\sim 50$ & 2.6 \\
\hline Buffer & 0 & $1: 85$ & 0 & () & $\sim 50$ & 5.0 \\
\hline Buffer & 0 & $1: 85$ & 0 & () & $\sim 50$ & 1.5 \\
\hline Buffer & 0 & $1: 85$ & 0 & () & $\sim 50$ & 1.6 \\
\hline
\end{tabular}

* Residual PTC of approximately $50 \%$ reflects virtually complete inhibitor neutralization in Mixture 1. Residual PTC of $5 \%$ or less shows that little or no inhibitor was neutralized in Mixture 1.

$\ddagger$ Final dilution of inhibitor in Mixture 1 .

$\$$ Calculated on the assumption that no inhibitor remained in the first incubation mixture. 


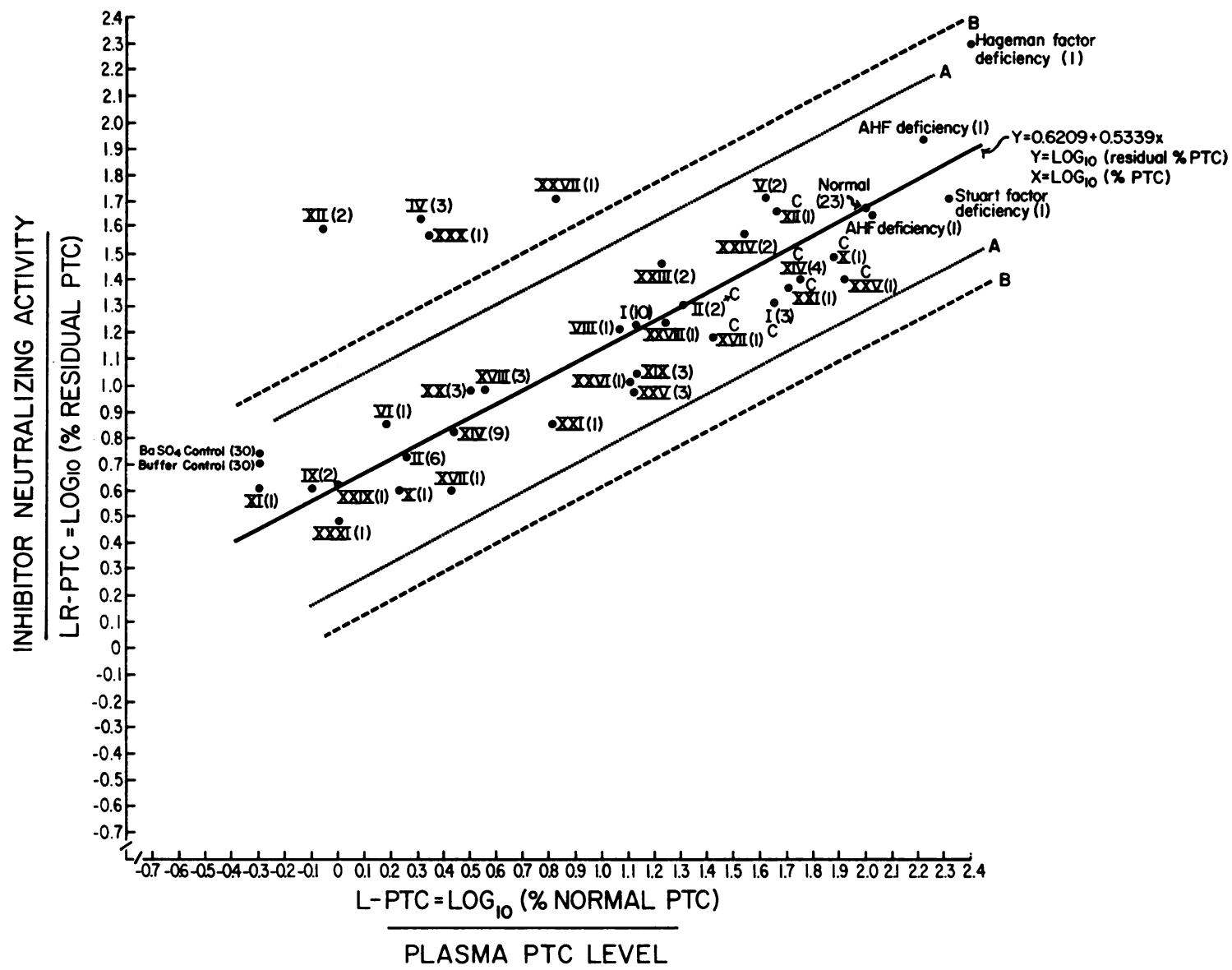

FIgURE 2 Log-log plot of plasma PTC level (L-PTC) against inhibitor-neutralizing capacity (LR-PTC). Curves labeled $\mathrm{A}$ are $99 \%$ confidence bands for kindred with two individuals; curves labeled B are $99 \%$ confidence bands for kindred with one individual. C denotes carriers; Roman numerals identify the kindred; and number in parentheses represents number of affected patients in the kindred. L-PTC is the initial concentration of PTC in plasma. LR-PTC is the residual PTC in Mixture 2 and reflects the amount of PTC-inhibitor neutralized in Mixture 1, which is related to inhibitor-neutralizing material in the test plasma.

ance (mean square, 0.5598 ). The ratio of 21.5 , which has 24 and $38^{\circ}$ of freedom, is significant at $P<0.01$.

Inhibitor-neutralizing capacity of normal plasma and other materials containing PTC. It can be seen in Fig. 2 that the mean PTC levels of 23 normal individuals was $100 \% \quad(\mathrm{~L}-\mathrm{PTC}=2)$. These plasmas also neutralized approximately $100 \%$ of added inhibitor as reflected by a residual PTC level in the second incubation mixture approaching $50 \%$ of normal (LR-PTC $=\sim 1.7$ ). The amount of inhibitor-neutralizing activity in AHF and Stuart factor deficient plasmas was similar to that found in normal plasma. Hageman factor deficient plasma, however, seemed to neutralize more inhibitor than expected on the basis of PTC level. Although not shown in Fig. 2, additional experiments indicated that other materials containing PTC activity, including normal serum, the eluate from $\mathrm{BaSO}_{4}$-adsorption, and PTC fractions, also contained inhibitor-neutralizing activity in the expected amounts.

Inhibitor-neutralizing capacity of plasma from patients with severe, moderate, and mild PTC deficiency. When inhibitor-neutralizing activity is plotted against plasma PTC level, the hemophilia $\mathrm{B}$ kindred may be divided into two distinct groups (Fig. 2).

The first group (Group 1) falls within the $99 \%$ confidence bands, and consists of 56 patients from 21 kindred. The major characteristic of this group is that there is a clear relationship between 
the PTC clotting activity and inhibitor-neutralizing activity; i.e., inhibitor-neutralizing activity is decreased in proportion to the diminished PTC clotting activity. In this respect, plasma from Group 1 resembles the $\mathrm{BaSO}_{4}$ and buffer controls.

The second group (Group 2) consists of seven patients in the four "outlier" kindred falling outside the $99 \%$ confidence bands. The major characteristic of Group 2 is that their PTC-inhibitorneutralizing activity is equivalent to normal and is clearly out of proportion to their reduced plasma PTC level.

Carriers from Group 1 have PTC levels approaching $50 \%$ of normal and, as expected, their neutralizing activity is less than normal. The only carrier from an "outlier" kindred available for study was carrier XII. Her inhibitor-neutralizing activity is equivalent to normal and greater than that of carriers from pedigrees with reduced inhibitor-neutralizing activity.

It is of considerable interest that mild, moderate, and severe forms of PTC deficiency are found in both groups of hemophilia B. Therefore. it is not possible to distinguish the two groups of PTC deficiency on the basis of clinical findings and PTC assays alone.

\section{DISCUSSION}

It is generally known that patients with hemophilia B can be classified as mild, moderate, or severe on the basis of clinical symptoms and plasma PTC levels. We have further subdivided these patientst into two groups: (1) those patients whose plasma contains material capable of neutralizing normal quantities of specific PTC-inhibitor and, (2) those whose plasma is deficient in inhibitor-neutralizing material.

Previous work in our laboratory strongly suggests that the specific PTC-inhibitor used in these experiments is an antibody which is neutralized by PTC or PTC-like material (1-3). Thus, the presence of inhibitor-neutralizing activity in plasma reflects the presence of either normal PTC or material serologically related to $\operatorname{PTC}(3,4)$.

We observed that the plasma in about $10 \%$ of the hemophilia B patients had as much inhibitorneutralizing activity as normal plasma even though the PTC clotting activity was markedly reduced. This suggests that the genetic mutation in these patients leads to an abnormally structured PTC molecule which is not fully effective as a procoagulant but retains the ability to neutralize a PTCinhibitor. It seems likely that the patient described by Fantl et al. would also fit into this category of hemophilia $B(4)$. In this respect. some hemophilia $\mathrm{B}$ patients may be analogous to $\mathrm{CRM}^{+}$ (cross-reacting material) mutants described in Neurospora and bacteria (9). It has been shown that in some mutant strains of Neurospora, the enzymatic activity of tryptophan synthetase cannot be detected. However, by immunochemical techniques, it has been found that most of these mutants produce normal levels of a protein serologically related to tryptophan synthetase, i.e., the tryptophan synthetase "deficiency" is not due to an absence of tryptophan sinthetase molecules but rather to the presence of structurally altered molecules lacking enzymatic activity. The mutation responsible for $\mathrm{CRM}^{+}$mutants must, therefore. affect enzyme structure rather than the rate of enzyme formation. On the other hand, some mutant strains of Neurospora, with deficient tryptophan synthetase activity, are $\mathrm{CRM}^{-}$, i.e., such mutants are not only deficient in enzymatic activity but are also lacking in antigenic activity (9). Perhaps in these cases the mutation results in an actual decreased production of enzyme molecules.

It is tempting to speculate that the group of hemophilia B patients with absent or decreased inhibitor-neutralizing activity is analogous to the $\mathrm{CRM}^{-}$mutants described in lower organisms. However, the inhibitor-neutralizing activity was not completely absent in some of the mild and moderately affected patients but rather decreased in proportion to the I'TC clotting activity. It does seem likely, however, that in the group of hemophilia $B$ patients with absent or decreased inhibitor-neutralizing activity, a mutation results in absent or decreased production or PTC. Whether the PTC produced in some patients of this group is structurally normal or abnormal cannot be answered on the basis of present data. It is interesting to note, however, that in control studies, materials lacking PTC (buffer and $\mathrm{BaSO}_{4}$-adsorbed normal plasma) are also deficient in inhibitor-neutralizing activity. Further evidence to suggest that the patients with decreased inhibitor-neutralizing activity may be deficient in PTC-like protein has been previously reported from our laboratory (2). We found that a patient who de- 
veloped a specific PTC-inhibitor after transfusions of normal plasma that contained PTC did not do so when transfused with plasma obtained from hemophilia B patients whose plasma contained no detectable inhibitor-neutralizing activity.

Hougie and Twomey have recently described a hemophilia B variant (hemophilia $\mathbf{B}_{\mathbf{M}}$ ) whose plasma gave a prolonged prothrombin time when tested with ox brain $(10,11)$. Whether this variant, which is genetically determined, is analogous to one of the hemophilia B types described in this paper is not known, since none of the prothrombin time tests performed on our patients has been done with ox brain.

Carriers with hemophilia $B$ should have approximately $50 \%$ PTC clotting activity. Our results, except for minimal overlapping, support this prediction. One would expect, however, that carriers from pedigrees with normal inhibitorneutralizing activity could be differentiated from carriers related to pedigrees with decreased inhibitor-neutralizing activity. Our results are in keeping with this expectation, and support our concept that the ability to neutralize a specific PTC-inhibitor is an inherited trait.

There was considerable quantitative variation in the PTC clotting activity as measured by the onestage PTC assay between kindred of both types of hemophilia B. Extensive pedigree studies failed to show family relationships between the separate kindred. It is possible that each kindred originated by independent mutation and represents a somewhat different intracistronic abnormality. It is conceivable that a large number of mutational sites exist at the PTC locus on the X-chromosome, and that each mutation results in a different type of PTC abnormality.

The reported data have withstood repeated laboratory testing and critical statistical evaluation. Furthermore, there was no overlap between data obtained from kindred with decreased inhibitorneutralizing activity and the four kindred with normal quantities of this material. Thus, it appears that hemophilia B may be subdivided on a basis other than plasma PTC levels and clinical severity. In this respect, hemophilia $B$ resembles some other hereditary disorders, such as other hemophilioid diseases, ovalocytosis, hemoglobinopathy, and G-6-PD deficiency, which, although clinically similar, have been shown to be genetically different.

\section{ACKNOWLEDGMENTS}

We are indebted to Dr. John B. Graham, Department of Pathology, for invaluable suggestions. The technical assistance of Mrs. Gwendolyn Gross, Mrs. Doris Sparrow, and $\mathrm{Mr}$. Bruce Beveridge is gratefully acknowledged.

Supported in part by U. S. Public Health Service grants HE- 06350 and GM-12868 of the National Institutes of Health and $U$. S. Public Health grant FR-46 from the General Clinical Research Centers Branch of the Division of Research Facilities and Resources.

\section{REFERENCES}

1. Roberts, H. R. 1964. A study of acquired inhibitor to blood clotting factor IX. Federation Proc. 23: 576. (Abstr.)

2. Roberts, H. R., G. P. Gross, W. P. Webster, I. I. Dejanov, and G. D. Penick. 1966. Acquired inhibitors of plasma factor IX: A study of their induction, properties, and neutralization. Am. J. Med. Sci. 251: 43.

3. McLester, IV. D., H. R. Roberts, and R. H. Wagner. 1965. Use of an immunosorbent technique in the study of a PTC inhibitor: A new method for the investigation of blood coagulation. J. Lab. Clin. Med. 66: 682.

4. Fantl, P., R. J. Sawers, and A. G. Marr. 1956. Investigation of a haemorrhagic disease due to betaprothromboplastin deficiency complicated by a specific inhibitor of thromboplastin formation. Australasian Ann. Med. 5: 163.

5. Langdell, R. D., R. H. Wagner, and K. M. Brinkhous. 1953. Effect of antihemophilic factor on one-stage clotting tests: a presumptive test for hemophilia and a simple one-stage antihemophilic factor assay procedure. I. Lab. Clin. Med. $41: 637$.

6. Hougie, C., E. MI. Barrow, and J. B. Graham. 1957. Stuart clotting defect. I. Segregation of an hereditary hemorrhagic state from the heterogeneous group heretofore called "Stable Factor" (SPCA, proconvertin, Factor VII) deficiency. J. Clin. Invest. 36: 485.

7. Roberts, H. R., M. B. Scales, J. T. Madison, W. P. Webster, and G. D. Penick. 1965. A clinical and experimental study of acquired inhibitors to Factor VIII. Blood. 26: 805.

8. Barrow, E. M., W. R. Bullock, and J. B. Graham. 1960. A study of the carrier state for plasma thromboplastin component (PTC, Christmas factor) deficiency, utilizing a new assay procedure. J. Lab. Clin. Med. 55: 936.

9. Bonner, D. M. 1961. Heredity. Foundations of Modern Biology Series. Prentice-Hall, Inc., Englewood Cliffs, N. J. 30.

10. Hougie, C., and J. J. Twomey. 1967. Haemophilia $B_{M}$ : A new type of factor-IX deficiency. Lancet. 1: 698.

11. Twomey, J. J., and C. Hougie. 1967. Hemophilia $B_{M}$ : A new type of Christmas disease. J. Clin. Invest. 46: 1125. (Abstr.) 